\title{
Phong cách học tập tiếng Trung Quốc của sinh viên Việt Nam: Nghiên cứu trường hợp sinh viên khoa tiếng Trung, Trường Đại học Sư phạm Thành phố Hồ Chí Minh \\ Vietnamese students' Chinese learning styles: A case study of students of department of Chinese, Ho Chi Minh City University of Education
}

\author{
Lưu Hớn Vũ ${ }^{1^{*}}$ \\ ${ }^{1}$ Trường Đại học Ngân hàng Thành phố Hồ Chí Minh, Việt Nam \\ *Tác giả liên hệ, Email: luuhonvu@gmail.com
}

THÔNG TIN

DOI: $10.46223 / \mathrm{HCMCOUJS}$. soci.vi.16.1.1346.2021

Ngày nhận: 09/12/2020

Ngày nhận lại: 05/04/2021

Duyệt đăng: 22/03/2021

Tù khóa:

phong cách học tập, tiếng Trung Quốc, Trường Đại học Sư phạm Thành phố Hồ Chí Minh

Keywords:

learning styles, Chinese, Ho Chi Minh City University of Education

\section{TÓM TẮT}

Bài viết nghiên cứu phong cách học tập tiếng Trung Quốc của sinh viên khoa tiếng Trung, Trường Đại học Sư phạm Thành phố Hồ Chí Minh. Trên cơ sở lí thuyết về phong cách học tập của Reid (1984), chúng tôi tiến hành khảo sát bằng bảng hỏi với 250 sinh viên. Kết quả cho thấy các loại phong cách học tập đều thuộc cấp độ phong cách học tập chính, trong đó phong cách học tập thính giác là phong cách học tập được yêu thích nhất. Giới tính không ảnh hưởng đến phong cách học tập của sinh viên. Song, thời gian học tập có ảnh hưởng đến phong cách học tập loại thính giác của sinh viên, vùng miền có ảnh hưởng đến phong cách học tập loại cá nhân của sinh viên. Phong cách học tập loại cá nhân và loại nhóm có ảnh hưởng đến kết quả học tập của sinh viên.

ABSTRACT
This article studies the Chinese learning styles of students
of the Department of Chinese at Ho Chi Minh City University
of Education. Based on Reid's learning style theory (1984), we
conducted a questionnaire survey with 250 students. The results
show that students' learning styles all belong to the main
learning style level, in which students prefer the auditory
learning style. Gender does not affect students' learning styles.
However, Chinese studying time affects the auditory learning
style; regions affect the individual learning style. The individual
learning style and the group learning style affect students'
academic performance.

\section{1. Đặt vấn đề}

Phong cách học tập (learning styles) là thuật ngữ được Thelen đưa ra vào năm 1954. Kể từ đó đến nay, phong cách học tập đã trở thành một trong những vấn đề nghiên cứu quan trọng của các lĩnh vực giáo dục học, tâm lí học và ngôn ngữ học, được xem là "nền tảng chân chính của giáo dục hiện đại” (Tan, 1995, p. 12). Keefe (1979) định nghĩa phong cách học tập là phương thức học tập tương đối ổn định của người học, phản ánh cách thức người học lĩnh hội thông tin, tương tác 
và phản ứng với môi trường học tập. Theo Gass và Selinker (2008), phong cách học tập là khuynh hướng của cá thể người học khi tiếp nhận, gia công và lưu trữ thông tin. Đây là một trong những nhân tố chủ yếu dẫn đến sự khác biệt cá thể ở người học ngoại ngữ (Ellis, 1994).

Nghiên cứu về phong cách học tập của người học tiếng Trung Quốc phát triển tương đối muộn. Mãi đến những năm đầu của thế kỉ XXI mới xuất hiện các công trình nghiên cứu về vấn đề này, như các nghiên cứu của Chen (2015), Cheng (2014), Wang (2017), Wei (2020), Wu (2009), Zhang (2014), ... Song, trong các nghiên cứu mà chúng tôi thu thập được, thành quả nghiên cứu về phong cách học tập tiếng Trung Quốc của sinh viên Việt Nam vẫn còn rất hạn chế.

Trong khuôn khổ bài viết này, chúng tôi muốn tìm câu trả lời cho ba vấn đề sau: Thứ nhất, đặc điểm phong cách học tập tiếng Trung Quốc của sinh viên Việt Nam như thế nào? Thứ hai, các nhân tố cá thể (giới tính, thời gian học tập, vùng miền) có ảnh hưởng như thế nào đến phong cách học tập tiếng Trung Quốc? Thứ ba, mối quan hệ giữa kết quả học tập với phong cách học tập tiếng Trung Quốc như thế nào?

\section{Cơ sở lý luận}

Reid (1984) cho rằng, con người học tập thông qua những cảm quan khác nhau, mỗi người đều có những sở thích khác nhau về cảm quan học tập và phương thức học tập, ví dụ có người thích học bằng "mắt", có người thích học bằng "tai”, có người thích học theo nhóm, có người thích học một mình. Vì vậy, Reid đã chia phong cách học tập thành sáu loại như sau (xem Bảng 1):

\section{Bảng 1}

Sáu loại phong cách học tập của Reid

\begin{tabular}{|c|l|}
\hline Phong cách học tập & \multicolumn{1}{c|}{ Định nghĩa và đặc điểm } \\
\hline $\begin{array}{c}\text { Loại thị giác } \\
\text { (visual) }\end{array}$ & $\begin{array}{l}\text { Người học tiếp nhận thông tin thông qua các kích thích thị giác. Nếu tài } \\
\text { liệu trực quan, sinh động, sẽ hình thành những hình ảnh rõ ràng trong não } \\
\text { người học, song nếu không có sự hồ trợ của các tài liệu thị giác, người học } \\
\text { sẽ khó ghi nhớ. Người học thuộc loại này không thích hợp với các hình } \\
\text { thức giảng dạy như đối thoại, giải thích bằng miệng. }\end{array}$ \\
\hline $\begin{array}{c}\text { Loại thính giác } \\
\text { (auditory) }\end{array}$ & $\begin{array}{l}\text { Người học tiếp nhận thông tin thông qua các kích thích thính giác. Người } \\
\text { học thuộc loại này thích hợp với các hình thức giảng dạy như giải thích } \\
\text { băng miệng, thảo luận, nhưng không thích hợp với việc xử lí tài liệu bằng } \\
\text { thị giác. }\end{array}$ \\
\hline $\begin{array}{c}\text { Loại xúc giác } \\
\text { (tactile) }\end{array}$ & $\begin{array}{l}\text { Người học thích được tiếp cận vấn đề thông qua đôi tay, như các hoạt động } \\
\text { thực hành với các vật liệu trong phòng thí nghiệm, xây dựng và xử lí mô hình. }\end{array}$ \\
\hline $\begin{array}{c}\text { Loại vận động } \\
\text { (kinesthetic) }\end{array}$ & $\begin{array}{l}\text { Người học thích được tiếp cận vấn đề thông qua các trải nghiệm vận động } \\
\text { cơ thể của bản thân trên lớp, như các trò chơi, đóng vai trên lớp. Người học } \\
\text { thuộc loại này thường cảm thấy khó khăn, khổ sở khi phải ngồi lâu một } \\
\text { chô̂. }\end{array}$ \\
\hline $\begin{array}{c}\text { Loại nhóm } \\
\text { (group) }\end{array}$ & $\begin{array}{l}\text { Người học thích học cùng người khác, rất xem trọng việc giao lưu, hợp tác } \\
\text { với người khác. }\end{array}$ \\
\hline $\begin{array}{c}\text { Loại cá nhân } \\
\text { (individual) }\end{array}$ & $\begin{array}{l}\text { Người học thích học một mình, cho rằng học một mình có hiệu quả hơn } \\
\text { học với người khác. }\end{array}$ \\
\hline
\end{tabular}

Nguồn: Tổng hợp của tác giả 
Với Max $=25$ và Min = 0 , Reid (1987) chia phong cách học tập làm ba cấp độ: phong cách học tập chính (major learning style) có Mean từ 13.5 đến 25, phong cách học tập phụ (minor learning style) có Mean từ 11.5 đến 13.49, phong cách học tập tiêu cực (negative learning style) có Mean từ 0 đến 11.49 .

\section{Phương pháp nghiên cứu}

\subsection{Khách thể nghiên cúu}

Tham gia khảo sát là 250 sinh viên khoa tiếng Trung, Trường Đại học Sư phạm Thành phố Hồ Chí Minh (HCMUE). Trong đó, có 20 sinh viên nam (chiếm tỉ lệ $8 \%$ ) và 230 sinh viên nữ (chiếm tỉ lệ 92\%); có 46 sinh viên năm thứ hai (chiếm tỉ lệ 18.4\%), 131 sinh viên năm thứ ba (chiếm tỉ lệ 52.4\%) và 73 sinh viên năm thứ tư (chiếm tỉ lệ $29.2 \%$ ); có 23 sinh viên đến từ các tỉnh, thành miền Bắc (chiếm tỉ lệ $9.2 \%$ ), 74 sinh viên đến từ các tỉnh, thành miền Trung (chiếm tỉ lệ $29.6 \%$ ) và 153 sinh viên đến từ các tỉnh, thành miền Nam (chiếm tỉ lệ 61.2\%).

\subsection{Công cụ thu thập dĩ liệu}

Chúng tôi sử dụng công cụ bảng hỏi để khảo sát phong cách học tập tiếng Trung Quốc của sinh viên khoa tiếng Trung Quốc HCMUE. Bảng khảo sát của chúng tôi được thiết kế dựa trên Bảng khảo sát phong cách học tập (Perceptual Learning Style Preference Questionnaire) của Reid (1984). Đây là bảng khảo sát được sử dụng rộng rãi trong nghiên cứu thụ đắc ngôn ngữ thứ hai, vì phạm vi được đề cập rộng, số lượng câu hỏi vừa phải (Wintergerst, DeCapua, \& Verna, 2003).

Bảng khảo sát gồm 30 câu, trong đó các câu Q6, Q10, Q12, Q24, Q29 là các câu hỏi về phong cách học tập loại thị giác, các câu Q1, Q7, Q9, Q17, Q20 là các câu hỏi về phong cách học tập loại thính giác, các câu Q11, Q14, Q16, Q22, Q25 là các câu hỏi về phong cách học tập loại xúc giác, các câu Q2, Q8, Q15, Q19, Q26 là các câu hỏi về phong cách học tập loại vận động, các câu Q3, Q4, Q5, Q21, Q23 là các câu hỏi về phong cách học tập loại nhóm, các câu Q13, Q18, Q27, Q28, Q30 là các câu hỏi về phong cách học tập loại cá nhân. Các câu trong bảng khảo sát đều sử dụng thang đo năm bậc của Likert từ "hoàn toàn không đồng ý" đến "hoàn toàn đồng ý".

\subsection{Quá trình khảo sát}

Chúng tôi tiến hành khảo sát vào tháng 11 năm 2020 tại khoa tiếng Trung HCMUE. Trước khi phát phiếu khảo sát, chúng tôi thông báo với sinh viên kết quả điều tra này không ảnh hưởng đến kết quả học tập của sinh viên, hi vọng sinh viên căn cứ vào tình hình thực tế của bản thân trả lời đầy đủ tất cả các câu hỏi có trong phiếu.

Chúng tôi phát ra 250 phiếu, thu vào 250 phiếu, tỉ lệ thu vào $100 \%$. Tất cả các phiếu thu vào đều là phiếu hợp lệ, sinh viên trả lời đầy đủ tất cả các câu hỏi có trong phiếu, đạt tỉ lệ $100 \%$.

\subsection{Công cụ phân tích dĩ liệu}

Chúng tôi sử dụng phần mềm SPSS (phiên bản 25.0) để thống kê, phân tích số liệu mà chúng tôi thu thập được. Trong bài viết này, chúng tôi sử dụng SPSS trong các thống kê mô tả, kiểm định giả thuyết về trị trung bình của hai tổng thể - trường hợp mẫu độc lập (Independent - samples $\mathrm{T}$ test), kiểm định phương sai một nhân tố (oneway ANOVA) và phân tích tương quan Pearson.

\section{Thảo luận kết quả}

\section{1. Đặc điểm phong cách học tập}

Đặc điểm phong cách học tập tiếng Trung Quốc của sinh viên khoa tiếng Trung HCMUE như sau (xem Bảng 2): 


\section{Bảng 2}

Đặc điểm phong cách học tập tiếng Trung Quốc

\begin{tabular}{|l|c|c|}
\hline \multicolumn{1}{|c|}{ Phong cách học tập } & Mean & SD \\
\hline Loại thị giác & 18.080 & 4.272 \\
\hline Loại thính giác & 20.240 & 2.654 \\
\hline Loại xúc giác & 18.772 & 3.278 \\
\hline Loại vận động & 20.100 & 3.016 \\
\hline Loại nhóm & 19.352 & 3.481 \\
\hline Loại cá nhân & 16.620 & 4.597 \\
\hline
\end{tabular}

Nguồn: Kết quả xử lí số liệu điều tra

Bảng 2 cho thấy, các loại phong cách học tập của sinh viên tham gia khảo sát đều có Mean $>13.5$ với độ lệch chuẩn SD tương đối thấp, đều thuộc cấp độ phong cách học tập chính; thứ tự từ cao đến thấp của sáu loại phong cách học tập là loại thính giác $>$ loại vận động $>$ loại nhóm $>$ loại xúc giác $>$ loại thị giác $>$ loại cá nhân. Có thể thấy, sinh viên thích sử dụng nhiều phong cách học tập khác nhau trong học tập tiếng Trung Quốc, trong đó ba phong cách học tập sinh viên thích nhất là phong cách học tập loại thính giác, phong cách học tập loại vận động và phong cách học tập loại nhóm, sinh viên không thích nhất là phong cách học tập loại cá nhân.

Kết quả này có những điểm tương đồng và dị biệt với kết quả nghiên cứu của Fang (2013) và Yin (2019) về phong cách học tập tiếng Trung Quốc của sinh viên Thái Lan và sinh viên Indonesia. Sinh viên Việt Nam cũng giống như sinh viên Thái Lan đều thích nhất là phong cách học tập loại thính giác, đều không thích nhất là phong cách học tập loại thị giác và loại cá nhân. Sinh viên Việt Nam và sinh viên Indonesia đều thích nhất là phong cách học tập loại thính giác và loại vận động, đều không thích nhất là phong cách học tập loại cá nhân.

\subsection{Anh hưởng của các nhân tố cá thể đối với phong cách học tập tiếng Trung Quốc}

4.2.1. Anh hưởng của giới tính đối với phong cách học tập tiếng Trung Quốc

Trong số sinh viên tham gia khảo sát, có 20 sinh viên nam (chiếm tỉ lệ $8 \%$ ) và 230 sinh viên nữ (chiếm tỉ lệ 92\%). Đặc điểm phong cách học tập tiếng Trung Quốc của sinh viên nam và sinh viên nữ như sau (xem Bảng 3):

\section{Bảng 3}

Đặc điểm phong cách học tập tiếng Trung Quốc theo giới tính

\begin{tabular}{|c|c|c|c|c|c|}
\hline Phong cách học tập & Giới tính & Mean & SD & $\mathbf{t}$ & $\mathbf{p}$ \\
\hline \multirow{2}{*}{ Loại thị giác } & Nam & 18.90 & 2.845 & \multirow{2}{*}{0.895} & \multirow{2}{*}{0.372} \\
\hline & Nữ & 18.01 & 4.371 & & \\
\hline \multirow{2}{*}{ Loại thính giác } & Nam & 19.85 & 2.581 & \multirow{2}{*}{-0.684} & \multirow{2}{*}{0.494} \\
\hline & Nữ & 20.27 & 2.663 & & \\
\hline \multirow{2}{*}{ Loại xúc giác } & Nam & 18.30 & 4.079 & \multirow{2}{*}{-0.548} & \multirow{2}{*}{0.590} \\
\hline & Nữ & 18.81 & 3.207 & & \\
\hline Loại vận động & Nam & 19.75 & 3.669 & -0.540 & 0.589 \\
\hline
\end{tabular}




\begin{tabular}{|l|c|c|c|c|c|}
\hline Phong cách học tập & Giới tính & Mean & SD & t & p \\
\hline \multirow{2}{*}{ Loại nhóm } & Nữ & 20.13 & 2.960 & & \\
\cline { 1 - 4 } \multirow{2}{*}{ Loại cá nhân } & Nam & 19.20 & 2.505 & \multirow{2}{*}{-0.272} & \multirow{2}{*}{0.788} \\
\cline { 2 - 4 } & Nữ & 19.37 & 3.557 & & \multirow{2}{*}{-0.425} \\
\cline { 2 - 4 } & Nam & 16.20 & 5.483 & \multirow{2}{*}{0.671} \\
\cline { 2 - 4 } & Nữ & 16.66 & 4.524 & & \\
\hline
\end{tabular}

Nguồn: Kết quả xử lí số liệu điều tra

Bảng 3 cho thấy, các loại phong cách học tập của sinh viên nam và sinh viên nữ đều có Mean > 13.5 với độ lệch chuẩn SD tương đối thấp, đều thuộc cấp độ phong cách học tập chính. Thứ tự từ cao đến thấp của sáu loại phong cách học tập của sinh viên nam là loại thính giác > loại vận động $>$ loại nhóm $>$ loại thị giác $>$ loại xúc giác $>$ loại cá nhân, và của sinh viên nữ là loại thính giác $>$ loại vận động $>$ loại nhóm $>$ loại xúc giác $>$ loại thị giác $>$ loại cá nhân. Điều này cho thấy, có sự khác biệt về thứ tự mức độ yêu thích phong cách học tập của sinh viên nam và sinh viên nữ. Sinh viên nam thích phong cách học tập loại thị giác hơn loại xúc giác, sinh viên nữ thì ngược lại.

Kết quả kiểm định giả thuyết về trị trung bình của hai tổng thể - trường hợp mẫu độc lập ở các loại phong cách học tập giữa sinh viên nam và sinh viên nữ đều không có sự khác biệt có ý nghĩa $(\mathrm{p}>0.05)$. Điều này cho thấy, giới tính không ảnh hưởng đến phong cách học tập tiếng Trung Quốc của sinh viên. Kết quả này giống với kết quả nghiên cứu của Yang (2012) và Yin (2019), không giống với kết quả nghiên cứu của Chen (2015), Wei (2020).

\subsubsection{Anh hương của thời gian học tập đối với phong cách học tập tiếng Trung Quốc}

Trong số sinh viên tham gia khảo sát, có 46 sinh viên năm thứ hai (chiếm tỉ lệ 18.4\%), 131 sinh viên năm thứ ba (chiếm tỉ lệ $52.4 \%$ ) và 73 sinh viên năm thứ tư (chiếm tỉ lệ $29.2 \%$ ). Mức độ các loại phong cách học tập tiếng Trung Quốc của sinh viên thuộc ba nhóm trên như sau (xem Bảng 4):

\section{Bảng 4}

Đặc điểm phong cách học tập tiếng Trung Quốc theo thời gian học tập

\begin{tabular}{|c|c|c|c|c|c|}
\hline Phong cách học tập & Cấp lớp & Mean & SD & $\mathbf{F}$ & $\mathbf{p}$ \\
\hline \multirow{3}{*}{ Loại thị giác } & Năm 2 & 19.24 & 8.006 & \multirow{3}{*}{2.462} & \multirow{3}{*}{0.087} \\
\hline & Năm 3 & 18.01 & 2.767 & & \\
\hline & Năm 4 & 17.48 & 2.829 & & \\
\hline \multirow{3}{*}{ Loại thính giác } & Năm 2 & 21.04 & 2.538 & \multirow{3}{*}{6.879} & \multirow{3}{*}{0.001} \\
\hline & Năm 3 & 20.45 & 2.506 & & \\
\hline & Năm 4 & 19.36 & 2.776 & & \\
\hline \multirow{3}{*}{ Loại xúc giác } & Năm 2 & 18.50 & 3.161 & \multirow{3}{*}{0.410} & \multirow{3}{*}{0.664} \\
\hline & Năm 3 & 18.95 & 3.146 & & \\
\hline & Năm 4 & 18.63 & 3.596 & & \\
\hline Loại vận động & Năm 2 & 20.35 & 3.171 & 0.878 & 0.417 \\
\hline
\end{tabular}




\begin{tabular}{|c|c|c|c|c|c|}
\hline Phong cách học tập & Cấp lớp & Mean & SD & $\mathbf{F}$ & $\mathbf{p}$ \\
\hline & Năm 3 & 20.23 & 2.805 & & \\
\hline & Năm 4 & 19.71 & 3.277 & & \\
\hline \multirow{3}{*}{ Loại nhóm } & Năm 2 & 20.02 & 2.848 & \multirow{3}{*}{1.922} & \multirow{3}{*}{0.149} \\
\hline & Năm 3 & 18.96 & 3.661 & & \\
\hline & Năm 4 & 19.63 & 3.462 & & \\
\hline \multirow{3}{*}{ Loại cá nhân } & Năm 2 & 16.67 & 4.437 & \multirow{3}{*}{1.340} & \multirow{3}{*}{0.264} \\
\hline & Năm 3 & 17.00 & 4.474 & & \\
\hline & Năm 4 & 15.90 & 4.882 & & \\
\hline
\end{tabular}

Nguồn: Kết quả xử lí số liệu điều tra

Bảng 4 cho thấy, các loại phong cách học tập của sinh viên năm hai, sinh viên năm ba và sinh viên năm tư đều có Mean > 13.5 với độ lệch chuẩn SD tương đối thấp, đều thuộc cấp độ phong cách học tập chính. Thứ tự từ cao đến thấp của sáu loại phong cách học tập của sinh viên năm hai là loại thính giác $>$ loại vận động $>$ loại nhóm $>$ loại thị giác $>$ loại xúc giác $>$ loại cá nhân, của sinh viên năm ba là loại thính giác $>$ loại vận động $>$ loại nhóm $>$ loại xúc giác $>$ loại thị giác $>$ loại cá nhân, và của sinh viên năm tư là loại vận động $>$ loại nhóm $>$ loại thính giác $>$ loại xúc giác $>$ loại thị giác $>$ loại cá nhân. Điều này cho thấy, có sự khác biệt về thứ tự mức độ yêu thích phong cách học tập của sinh viên năm hai, sinh viên năm ba và sinh viên năm tư. Sinh viên năm hai thích phong cách học tập loại thị giác hơn loại xúc giác, sinh viên năm ba thì ngược lại. Sinh viên năm tư thích phong cách học tập loại vận động và loại nhóm hơn loại thính giác, sinh viên năm hai và sinh viên năm ba thì ngược lại.

Kết quả kiểm định phương sai một nhân tố ở các loại phong cách học tập giữa sinh viên năm hai, sinh viên năm ba và sinh viên năm tư cho thấy, có sự khác biệt có ý nghĩa $(\mathrm{p}<0.05)$ về phong cách học tập loại thính giác. Sinh viên năm hai yêu thích phong cách học tập loại thính giác hơn sinh viên năm ba và sinh viên năm tư. Điều này cho thấy, thời gian học tập có ảnh hưởng đến phong cách học tập tiếng Trung Quốc loại thính giác của sinh viên. Kết quả này không giống với kết quả nghiên cứu của Yang (2012) và Fang (2013).

\subsubsection{Anh hương của vùng miền đối với phong cách học tập tiếng Trung Quốc}

Trong số sinh viên tham gia khảo sát, có 23 sinh viên đến từ các tỉnh, thành miền Bắc (chiếm tỉ lệ 9.2\%), 74 sinh viên đến từ các tỉnh, thành miền Trung (chiếm tỉ lệ $29.6 \%$ ) và 153 sinh viên đến từ các tỉnh, thành miền Nam (chiếm tỉ lệ $61.2 \%$ ). Mức độ các loại phong cách học tập tiếng Trung Quốc của sinh viên thuộc ba nhóm trên như sau (xem Bảng 5):

\section{Bảng 5}

Đặc điểm phong cách học tập tiếng Trung Quốc theo vùng miền

\begin{tabular}{|l|c|c|c|c|c|}
\hline Phong cách học tập & Vùng miền & Mean & SD & F & p \\
\hline \multirow{2}{*}{ Loại thị giác } & Bắc & 17.52 & 2.695 & & \multirow{2}{*}{1.239} \\
\cline { 2 - 4 } & Trung & 17.55 & 2.785 & 0.291 \\
\cline { 2 - 4 } & Nam & 18.42 & 4.982 & & \\
\hline Loại thính giác & Bắc & 20.57 & 2.555 & 0.229 & 0.795 \\
\hline
\end{tabular}




\begin{tabular}{|c|c|c|c|c|c|}
\hline Phong cách học tập & Vùng miền & Mean & SD & $\mathbf{F}$ & $\mathbf{p}$ \\
\hline & Trung & 20.14 & 2.873 & & \\
\hline & Nam & 20.24 & 2.570 & & \\
\hline \multirow{3}{*}{ Loại xúc giác } & Bắc & 18.78 & 2.891 & \multirow{3}{*}{0.111} & \multirow{3}{*}{0.895} \\
\hline & Trung & 18.92 & 3.096 & & \\
\hline & Nam & 18.70 & 3.432 & & \\
\hline \multirow{3}{*}{ Loại vận động } & Bắc & 20.13 & 2.959 & \multirow{3}{*}{1.421} & \multirow{3}{*}{0.243} \\
\hline & Trung & 20.58 & 2.966 & & \\
\hline & Nam & 19.86 & 3.039 & & \\
\hline \multirow{3}{*}{ Loại nhóm } & Bắc & 20.43 & 3.422 & \multirow{3}{*}{1.989} & \multirow{3}{*}{0.139} \\
\hline & Trung & 19.65 & 3.692 & & \\
\hline & Nam & 19.05 & 3.361 & & \\
\hline \multirow{3}{*}{ Loại cá nhân } & Bắc & 14.57 & 4.110 & \multirow{3}{*}{4.823} & \multirow{3}{*}{0.009} \\
\hline & Trung & 15.92 & 4.730 & & \\
\hline & Nam & 17.27 & 4.485 & & \\
\hline
\end{tabular}

Nguồn: Kết quả xử lí số liệu điều tra

Bảng 5 cho thấy, các loại phong cách học tập của sinh viên đến từ các tỉnh, thành miền Bắc, sinh viên đến từ các tỉnh, thành miền Trung và sinh viên đến từ các tỉnh, thành miền Nam đều có Mean > 13.5 với độ lệch chuẩn SD tương đối thấp, đều thuộc cấp độ phong cách học tập chính. Thứ tự từ cao đến thấp của sáu loại phong cách học tập của sinh viên đến từ các tỉnh, thành miền Bắc là loại thính giác > loại nhóm $>$ loại vận động $>$ loại xúc giác $>$ loại thị giác $>$ loại cá nhân, của sinh viên đến từ các tỉnh, thành miền Trung là loại vận động $>$ loại thính giác $>$ loại nhóm $>$ loại xúc giác $>$ loại thị giác $>$ loại cá nhân, và của sinh viên đến từ các tỉnh, thành miền Nam là loại thính giác $>$ loại vận động $>$ loại nhóm $>$ loại xúc giác $>$ loại thị giác $>$ loại cá nhân. Điều này cho thấy, có sự khác biệt về thứ tự mức độ yêu thích phong cách học tập của sinh viên đến từ các vùng miền khác nhau. Sinh viên đến từ các tỉnh, thành miền Bắc thích phong cách học tập loại nhóm hơn loại vận động, sinh viên đến từ các tỉnh, thành miền Nam và miền Trung thì ngược lại. Sinh viên đến từ các tỉnh, thành miền Bắc và miền Nam đều thích phong cách học tập loại thính giác hơn loại vận động, sinh viên đến từ các tỉnh, thành miền Trung thì ngược lại.

Kết quả kiểm định phương sai một nhân tố ở các loại phong cách học tập giữa sinh viên đến từ các tỉnh, thành miền Bắc, sinh viên đến từ các tỉnh, thành miền Trung và sinh viên đến từ các tỉnh, thành miền Nam cho thấy có sự khác biệt có ý nghĩa $(\mathrm{p}<0.05)$ ở phong cách học tập loại cá nhân. Điều này cho thấy, vùng miền có ảnh hưởng đến phong cách học tập tiếng Trung Quốc loại cá nhân của sinh viên.

\subsection{Mối quan hệ giữa kết quả học tập và phong cách học tập tiếng Trung Quốc}

Chúng tôi sử dụng phương pháp phân tích tương quan Pearson để kiểm định mối tương quan giữa kết quả học tập và phong cách học tập tiếng Trung Quốc. Kết quả như sau (xem Bảng 6): 


\section{Bảng 6}

Phân tích tương quan giữa kết quả học tập và phong cách học tập tiếng Trung Quốc

\begin{tabular}{|c|c|c|c|c|c|c|}
\hline & $\begin{array}{c}\text { Loại thị } \\
\text { giác }\end{array}$ & $\begin{array}{c}\text { Loại thính } \\
\text { giác }\end{array}$ & $\begin{array}{c}\text { Loại xúc } \\
\text { giác }\end{array}$ & $\begin{array}{c}\text { Loại vận } \\
\text { động }\end{array}$ & Loại nhóm & $\begin{array}{c}\text { Loại cá } \\
\text { nhân }\end{array}$ \\
\hline $\begin{array}{c}\text { Kết quả học } \\
\text { tập }\end{array}$ & $\mathrm{r}=0.057$ & $\mathrm{r}=0.072$ & $\mathrm{r}=0.017$ & $\mathrm{r}=0.086$ & $\mathrm{r}=-0.144$ & $\mathrm{r}=0.158$ \\
$\mathrm{p}=0.371$ & $\mathrm{p}=0.258$ & $\mathrm{p}=0.792$ & $\mathrm{p}=0.175$ & $\mathrm{p}=0.022$ & $\mathrm{p}=0.012$ \\
\hline
\end{tabular}

Nguồn: Kết quả xử lí số liệu điều tra

Bảng 6 cho thấy, tồn tại mối tương quan thuận giữa kết quả học tập và phong cách học tập loại cá nhân $(\mathrm{p}<0.05)$ và mối tương quan nghịch giữa kết quả học tập và phong cách học tập loại nhóm $(\mathrm{p}<0.05)$. Qua đó cho thấy, sinh viên càng thích phong cách học tập loại cá nhân thì có kết quả học tập càng cao, sinh viên càng thích phong cách học tập loại nhóm thì có kết quả học tập càng thấp. Có thể nói, kết quả học tập chịu ảnh hưởng nhất định bởi phong cách học tập loại nhóm và loại cá nhân của sinh viên. Nói cách khác, sinh viên càng có khuynh hướng phong cách học tập loại cá nhân sẽ có kết quả học tập càng cao, sinh viên càng có khuynh hướng phong cách học tập loại nhóm sẽ có kết quả học tập càng thấp.

\section{Kết luận và khuyến nghị}

Các loại phong cách học tập tiếng Trung Quốc của sinh viên khoa tiếng Trung HCMUE đều thuộc cấp độ phong cách học tập chính, có thứ tự từ cao đến thấp là loại thính giác $>$ loại vận động $>$ loại nhóm $>$ loại xúc giác $>$ loại thị giác $>$ loại cá nhân. Song, có sự khác biệt thứ tự mức độ yêu thích các loại phong cách học tập giữa sinh viên nam và sinh viên nữ, giữa sinh viên năm hai, năm ba và sinh viên năm tư, giữa sinh viên đến từ các tỉnh, thành miền Bắc, miền Trung và miền Nam.

Nhân tố giới tính không ảnh hưởng đến phong cách học tập của sinh viên. Song, nhân tố thời gian học tập có ảnh hưởng đến phong cách học tập loại thính giác của sinh viên, nhân tố vùng miền có ảnh hưởng đến phong cách học tập loại cá nhân của sinh viên. Kết quả học tập chịu ảnh hưởng bởi phong cách học tập của sinh viên, sinh viên càng có khuynh hướng phong cách học tập loại cá nhân sẽ có kết quả học tập càng cao, sinh viên càng có khuynh hướng phong cách học tập loại nhóm sẽ có kết quả học tập càng thấp.

Trên cơ sở các kết quả nghiên cứu trên đây, chúng tôi xin đưa ra một số kiến nghị sau:

Thứ nhất, phong cách học tập loại thính giác là phong cách học tập sinh viên yêu thích nhất. Vì vậy, trong quá trình giảng dạy giảng viên nên chủ động sử dụng tiếng Trung Quốc, yêu cầu sinh viên đọc to khi đọc từ vựng, mẫu câu, bài khoá. Ngoài ra, giảng viên cũng nên cung cấp thêm các tài liệu kích thích thính giác của sinh viên, như các đoạn ghi âm bài khoá, hoặc bổ sung các tài liệu kết hợp cả thính giác và thị giác như các đoạn video clip có nội dung liên quan đến bài học..., qua đó phát huy hơn nữa sở trường thính giác của sinh viên.

Thứ hai, phong cách học tập loại thị giác là một trong những phong cách học tập không được sinh viên yêu thích. Điều này có thể sẽ ảnh hưởng đến việc phát triển các kĩ năng đọc, viết tiếng Trung Quốc của sinh viên. Vì vậy, trong quá trình giảng dạy giảng viên cần chú ý cách trình bày bài giảng trên bảng, đồng thời bổ sung thêm các tài liệu trực quan, sinh động (như hình ảnh, vật mô phỏng...) kích thích thị giác của sinh viên.

Thứ ba, phong cách học tập loại cá nhân là phong cách học tập không được sinh viên yêu thích nhất. Song, loại phong cách học tập này lại có ảnh hưởng đến kết quả học tập của sinh viên. 
Những sinh viên yêu thích phong cách học tập loại cá nhân là những sinh viên có kết quả học tập cao, còn những sinh viên yêu thích phong cách học tập loại nhóm lại là những sinh viên có kết quả học tập thấp. Đây là điều rất đáng được quan tâm. Xu hướng giáo dục hiện nay là đưa các hoạt động nhóm vào lớp học, nhằm tăng tính tích cực trong học tập của sinh viên, song đại đa số các bài đánh giá quá trình và kết thúc học phần đều yêu cầu cá nhân tự hoàn thành. Vì vậy, giảng viên cần kết hợp bài tập cá nhân và bài tập nhóm trong hoạt động giảng dạy và đánh giá kết quả học tập. Đồng thời, khuyến khích các sinh viên có phong cách học tập loại cá nhân tham gia các hoạt động nhóm, giúp đỡ các bạn trong nhóm để mọi người cùng tiến bộ.

\section{Tài liệu tham khảo}

Chen, T. X. (2015). Meiguo daxue Hanyu xuexizhe xuexi fengge qingxiangxing yanjiu [A study of learning preferences about CSL learners in American universities]. Comparative Education Review, 37(12), 16-23.

Cheng, Z. J. (2014). Jiyu SPSS ruanjian de Hanyu chu gaoji liuxuesheng xuexi fengge de duibi yanjiu [A comparative study of the learning styles of Chinese elementary and advanced foreign students based on SPSS software]. Modern Chinese (Language Research Edition), 12, 67-71.

Ellis, R. (1994). The study of second language acquisition. Oxford, UK: Oxford University Press.

Fang, M. (2013). Taiguo xuesheng Hanyu xuexi fengge ji qi xuexi xiaoguo yanjiu [A study on Thai students' Chinese learning styles and their learning effects] (Master's thesis). Shanghai Normal University, Shanghai, China.

Gass, S., \& Selinker, L. (2008). Second language acquisition: An introductory course (3rd ed.). New York, USA: Routledge.

Keefe, J. W. (1979). Learning style: An overview. In NASSP's student learning styles: Diagnosing and prescribing programs. Reston, VA: National Association of Secondary School Principals.

Reid, J. M. (1984). Perceptual learning style preference questionnaire. Laramie, WY: University of Wyoming, Department of English.

Reid, J. M. (1987). The learning style preferences of ESL students. TESOL Quarterly, 21(1), 87110.

Tan, D. L. (1995). Xuexi fengge lun [Learning style theory]. Nanjing, China: Jiangsu Education Press.

Thelen, H. (1954). Dynamics of groups at work. Chicago, IL: The University of Chicago Press.

Wang, X. Y. (2017). Hanyu xuexi fengge diaocha yanjiu - Yi Shanghai Jiaotong Daxue Hanyu guoji jiaoyu zhongxin liuxuesheng wei li [Investigation and research on Chinese learning style - A case study of international students of Shanghai Jiao Tong University Chinese International Education Center]. Modern Chinese (Academic Comprehensive Edition), 6, 142-146.

Wei, Z. Y. (2020). Zhongya Dongganzu liuxuesheng Hanyu ganzhi xuexi fengge de diaocha yu yanjiu - Yi Xibei Shifan Daxue wei li [Investigation and research on Chinese perceptual learning style of Dungan international students in Central Asia - A case study of Northwest Normal University] (Master's thesis). Northwest Normal University, Lanzhou, China.

Wintergerst, A. C., DeCapua, A., \& Verna, M. A. (2003). Conceptualizing learning style modalities for ESL/EFL students. System, 31(1), 85-106. 
Wu, S. N. (2009). Xiongyali xuesheng de xuexi fengge ji qi dui Hanyu ketang huodong pianhao de yingxiang [The learning style of Hungarian students and its influence on the preference of Chinese classroom activities]. International Chinese Language Education, 4, 51-60.

Yang, Z. L. (2012). Chenggong Hanyu xuexizhe de xuexi celue ji xuexi fengge yanjiu [A study of learning strategies and learning styles of successful Chinese learners] (Master's thesis). Shandong University, Jinan, China.

Yin, J. Z. (2019). Yinni lai Hua liuxuesheng Hanyu xuexi fengge yu chengji xiangguanxing yanjiu Yi Shanghai Waiguoyu Daxue wei li [The correlation research between Chinese learning styles and achievements of Indonesian students in China - Taking Shanghai International Studies University as an example] (Master's thesis). Shanghai International Studies University, Shanghai, China.

Zhang, L. (2014). Yidali zhongji Hanyu xuexizhe de xuexi fengge he jiaoxue qishi [Learning styles and teaching enlightenment of Italian intermediate Chinese learners]. Newsletter of the International Society for Chinese Language Teaching, 2, 9-11. 